\title{
Spin-Swapping Transport and Torques in Ultrathin Magnetic Bilayers
}

\author{
Hamed Ben Mohamed Saidaoui ${ }^{\dagger}$ and A. Manchon* \\ Physical Science and Engineering Division, King Abdullah University of Science and Technology (KAUST), \\ Thuwal 23955-6900, Kingdom of Saudi Arabia \\ (Received 11 November 2015; revised manuscript received 24 February 2016; published 12 July 2016)
}

\begin{abstract}
Planar spin transport in disordered ultrathin magnetic bilayers comprising a ferromagnet and a normal metal (typically used for spin pumping, spin Seebeck and spin-orbit torque experiments) is investigated theoretically. Using a tight-binding model that puts the extrinsic spin Hall effect and spin swapping on equal footing, we show that the nature of spin-orbit coupled transport dramatically depends on the ratio between the layer thickness $d$ and the mean free path $\lambda$. While the spin Hall effect dominates in the diffusive limit $(d \gg \lambda)$, spin swapping dominates in the Knudsen regime $(d \lesssim \lambda)$. A remarkable consequence is that spin swapping induces a substantial fieldlike torque in the Knudsen regime.
\end{abstract}

DOI: 10.1103/PhysRevLett.117.036601

Introduction.-Spin-orbit coupling is responsible for a wide variety of phenomena that have attracted a great amount of attention recently [1,2]. Among the most prominent phenomena, one can cite the inverse spin galvanic effect-i.e., the electrical generation of a nonequilibrium magnetization [3] — and the spin Hall effect [4,5] - i.e., the conversion of an unpolarized charge current to a pure spin current. The nature of the spin Hall effect has been scrutinized intensively recently due to its central role in spintronics. While the original theory was based on carrier scattering against extrinsic spin-orbit coupled impurities [5], the importance of the band structure's Berry curvature has recently been unveiled, producing large dissipationless (i.e., scattering independent) spin Hall effects [6]. Although the proper theoretical treatment of this effect in the diffusive limit continues to inspire debate [7], experiments tend to confirm the importance of intrinsic spin Hall effect in $4 d$ and $5 d$ transition metals $[8,9]$. In the opposite limit, i.e., when the system size is comparable to the mean free path (the so-called Knudsen regime), the nature of the spin Hall effect changes subtly as quantum and semiclassical size effects emerge [10]. An accurate description of spin-orbit coupled transport in this regime is crucial, as ultrathin normal metal-ferromagnet bilayers (e.g., $\mathrm{Pt} / \mathrm{NiFe}$, etc.) are now commonly used in spin pumping [11], spin Seebeck effect [12], and spin-orbit torque measurements $[13,14]$.

The limitations of current models of the spin Hall effect are best illustrated by the puzzles raised by spin-orbit torque experiments. In magnetic systems lacking inversion symmetry, spin-orbit coupling enables the electrical control of the magnetic order parameter [15-18]. This spin-orbit mediated torque has been observed in combinations of various materials involving heavy metals [13,14], oxides [19], and topological insulators [20]. Experimentally, the torque possesses two components, referred to as damping and fieldlike torques, which are even and odd in magnetization direction, respectively. Consensually, the damping torque is associated with the spin Hall effect occurring in the bulk of the heavy metal [21] [see Fig. 1(a)], while the fieldlike torque is usually attributed to the inverse spin galvanic effect induced by spin-orbit coupling at the interface with the ferromagnet $[15,16]$. Nonetheless, recent experiments suggest that the inverse spin galvanic effect might not be the sole origin of fieldlike torque in ultrathin magnetic bilayers. Indeed, (i) sizable fieldlike torques have been reported in systems where interfacial spin-orbit coupling is expected to be small [22], and (ii) significant modulation of the fieldlike torque has been obtained upon varying the heavy metal thickness [22-24]. While both observations contradict the inverse spin galvanic effect scenario, no alternative mechanism has been identified, despite intense theoretical efforts $[21,25,26]$.

In this Letter, we demonstrate theoretically that the nature of extrinsic spin-orbit coupled transport in disordered ultrathin magnetic bilayers dramatically depends on the transport regime. When the disorder is strong and the transport is diffusive, the spin Hall effect dominates, leading to a damping torque that agrees with the widely (a)

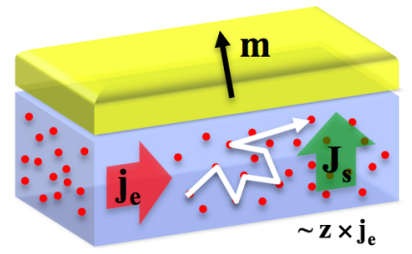

(b)

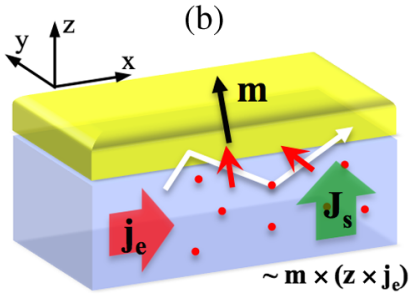

FIG. 1. Schematics of (a) spin Hall and (b) spin swapping effects in a bilayer composed of a normal metal (blue) and a ferromagnet (yellow) with magnetization $\mathbf{m}$ in the diffusive and Knudsen regimes, respectively. The charge current $\mathbf{j}_{e}$ is injected in the plane of the layers and results in a spin current $\mathbf{J}_{s}$ that flows perpendicularly to the interface. 
accepted physical picture [21]. In contrast, when disorder is weak and the system size is of the order of the carrier mean free path, spin swapping [27] becomes increasingly important, leading to a substantial fieldlike torque.

General principles. - Consider a metallic bilayer composed of a spin-orbit coupled normal metal and a ferromagnet without spin-orbit coupling (see Fig. 1). A current $\mathbf{j}_{e}$ is injected into the plane of the bilayer, exerting a torque on the ferromagnet. Disregarding the inverse spin galvanic effect, two spin-orbit coupled transport phenomena coexist. First, because of an intrinsic and/or extrinsic spin Hall effect in the normal metal, a spin current flows along the normal to the interface $\mathbf{z}$ with a spin polarization along $\left(\mathbf{z} \times \mathbf{j}_{e}\right)$. This results in a spin torque of the form $\sim \mathbf{m} \times\left[\left(\mathbf{z} \times \mathbf{j}_{e}\right) \times \mathbf{m}\right]$, which is of dampinglike form [21] (even in $\mathbf{m}$ ); see Fig. 1(a). In addition, electrons flowing in the ferromagnet acquire a spin polarization along $\mathbf{m}$ and may scatter towards the normal metal. Once in the normal metal, these electrons experience spin swapping: upon scattering on spin-orbit coupled impurities, they experience a spin-orbit field oriented normal to the scattering plane [i.e., along $\left.\left(\mathbf{z} \times \mathbf{j}_{e}\right)\right]$ and about which their spin precesses $[27,28]$. Upon this reorientation, a spin current polarized along $\mathbf{m} \times\left(\mathbf{z} \times \mathbf{j}_{e}\right)$ is injected into the ferromagnet and induces a fieldlike torque (odd in $\mathbf{m}$ ) —-see Fig. 1(b) —even in the absence of the inverse spin galvanic effect. Since these two effects operate in distinct disorder regimes- that is, the spin Hall effect necessitates strong disorder while spin swapping survives even for weak disorder [28]—the nature of the torque should dramatically change from one regime to the other.

Numerical results. - To investigate these effects quantitatively, we computed the spin transport in a magnetic bilayer using a tight-binding model $[28,29]$. The system is a two-dimensional square lattice connected laterally to external leads. The full Hamiltonian of the central system reads

$$
\begin{aligned}
\hat{H}= & \sum_{i, j, \sigma, \sigma^{\prime}}\left\{\left(\epsilon_{i j} \delta_{\sigma \sigma^{\prime}}+\frac{\Delta_{i j}}{2} \mathbf{m} \cdot \hat{\boldsymbol{\sigma}}_{\sigma \sigma^{\prime}}\right) \hat{c}_{i, j, \sigma}^{+} \hat{c}_{i, j, \sigma^{\prime}}+\text { H.c. }\right\} \\
& -\sum_{i, j, \sigma} t_{N}\left(\hat{c}_{i+1, j, \sigma}^{+} \hat{c}_{i, j, \sigma}+\hat{c}_{i, j+1, \sigma}^{+} \hat{c}_{i, j, \sigma}+\text { H.c. }\right) \\
& +i \sum_{i, j} t_{i, j-1}^{i-1, j}\left(\hat{c}_{i, j, \uparrow}^{+} \hat{c}_{i-1, j-1, \downarrow}-\hat{c}_{i, j, \downarrow}^{+} \hat{c}_{i-1, j-1, \uparrow}+\text { H.c. }\right) \\
& +i \sum_{i, j} t_{i-1, j-1}^{i, j}\left(\hat{c}_{i, j-1, \uparrow}^{+} \hat{c}_{i-1, j, \downarrow}-\hat{c}_{i, j-1, \downarrow}^{+} \hat{c}_{i-1, j, \uparrow}+\text { H.c. }\right) .
\end{aligned}
$$

Here, the first term at the right-hand side of Eq. (1) is the spin-independent on-site energy where $\epsilon_{i j}=\epsilon_{0}+\gamma_{i j}$, with $\epsilon_{0}$ being the onsite energy and $\gamma_{i j} \in[-\Gamma / 2, \Gamma / 2]$ a random potential of strength $\Gamma$ that introduces disorder in the system. The second term is the exchange interaction $\left(\equiv \Delta_{i j}\right)$ between the spin of the carriers and the local magnetic moment of direction $\mathbf{m}$ on site $(i, j)$. The third term in the Hamiltonian corresponds to the nearest neighbor hopping energy $\left(\equiv t_{N}\right)$. The last two terms are the next-nearest neighbor hopping parameters that account for the disorder-driven spin-orbit coupled scattering. The next-nearest neighbor hopping parameter reads $t_{i^{\prime}, j^{\prime}}^{i, j}=$ $i t_{N} \alpha\left(\epsilon_{i, j}-\epsilon_{i^{\prime}, j^{\prime}}\right)$, where $\alpha$ is the dimensionless spin-orbit coupling strength. The operator $\hat{c}_{i, j, \sigma}^{+}\left(\hat{c}_{i, j, \sigma}\right)$ creates (annihilates) a particle with spin $\sigma$ at the position $(i, j)$. This approach models the extrinsic spin Hall effect and spin swapping on equal footing, and one can tune the relative strength between the spin Hall effect and spin swapping by changing the disorder strength $\Gamma$ (and therefore the mean free path, $\lambda$ ) and the spin-orbit coupling strength $\alpha$ [28]. Notice that this spin-orbit coupling creates a random field along $\mathbf{y}$ and thereby relaxes the spin components in the $(\mathbf{x}, \mathbf{z})$ plane. Such a spin relaxation does not impact our numerical results significantly, as our system size remains smaller than the spin relaxation length.

Let us now consider the current-driven spin density in a two-dimensional bilayer in the $(\mathbf{x}, \mathbf{z})$ plane (see Fig. 1). The width of the ferromagnet (the normal metal) is $d_{F}=10 a$ $\left(d_{N}=20 a\right)$, and the length of the bilayer is $L=30 a$, where $a$ is the square lattice parameter. Figure 2(a) shows the nonequilibrium spin density profile along the bilayer width obtained for a strongly disordered system $(\Gamma=2.2 \mathrm{eV})$ and various spin-orbit coupling strengths, $\alpha$ [30]. The corresponding two-dimensional mapping is (a) $\Gamma=2.2 \mathrm{eV}$

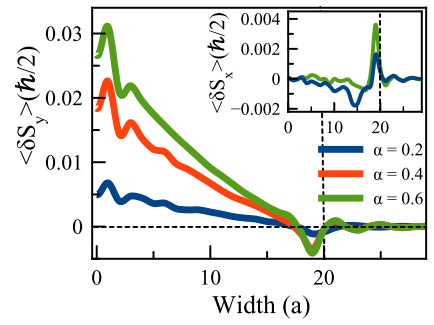

(c) $\Gamma=0.1 \mathrm{eV}$

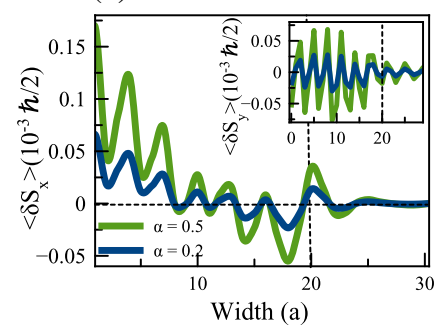

(b)

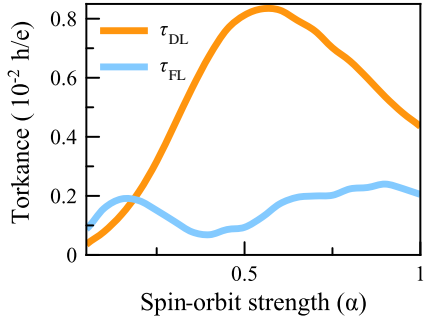

(d)

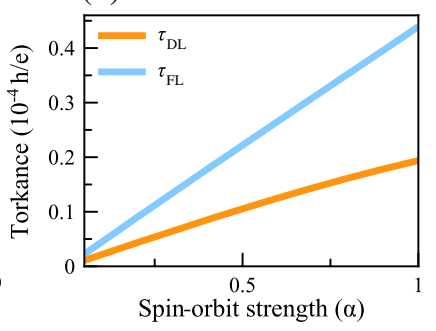

FIG. 2. (a),(c) Spin density profile along the magnetic bilayer width for strong $(\Gamma=2.2 \mathrm{eV})$ and weak disorder $(\Gamma=0.1 \mathrm{eV})$ regimes. The vertical dashed line separates the normal metal (left) from the ferromagnetic layer (right). The main panels (the insets) represent the largest (smallest) spin density component for various $\alpha$ 's. (b),(d) Corresponding spin torkance components as a function of $\alpha$. The parameters are $t_{N}=\epsilon_{0}=\Delta=1 \mathrm{eV}$ and $\mathbf{m}=\mathbf{z}$. 
shown in the Supplemental Material [31]. The spin density is mainly aligned along $\delta S_{y}$ (the main panel) and has a small $\delta S_{x}$ contribution (the inset). Remarkably, $\delta S_{y}$ smoothly accumulates over the layer width, while the small $\delta S_{x}$ component is confined at the interface. Notice that oscillations stemming from quantum coherence survive even for this amount of disorder, as no extrinsic quantum dephasing is introduced. These results indicate that $\delta S_{y}$ arises from the spin Hall effect, while $\delta S_{x}$ originates from spin swapping: the former extends over the layer width, whereas the latter only survives within a distance of the order of the mean free path (see also Fig. 8 in Ref. [28]).

The efficiency of the torque (torkance) exerted on the magnetic layer is defined $\boldsymbol{\tau}=(\Delta / G) \int d \boldsymbol{\Omega} \delta \mathbf{S} \times \mathbf{m}$ (where $\Omega$ is the volume of the magnet and $G$ is the conductance of the bilayer). To analyze its symmetry, we extract its fieldlike $\left(\tau_{\mathrm{FL}}\right)$ and dampinglike components $\left(\tau_{\mathrm{DL}}\right)$ such that $\boldsymbol{\tau}=\tau_{\mathrm{FL}} \mathbf{m} \times\left(\mathbf{z} \times \mathbf{j}_{e}\right)+\tau_{\mathrm{DL}} \mathbf{m} \times\left[\left(\mathbf{z} \times \mathbf{j}_{e}\right) \times \mathbf{m}\right]$. In a strongly disordered regime, the torkance is mostly dampinglike, $\tau_{\mathrm{DL}}>\tau_{\mathrm{FL}}$, but when reducing the spin-orbit strength, the spin Hall effect decreases and $\tau_{\mathrm{FL}}>\tau_{\mathrm{DL}}$, as illustrated in Fig. 2(b). This crossover occurs because spin relaxation, which is detrimental to spin swapping, decreases with $\alpha$, thereby enhancing spin swapping (see Fig. 8 in Ref. [28]). This region can be widened by decreasing the disorder strength, as shown in Fig. 3.

The case of weak disorder $(\Gamma=0.1 \mathrm{eV})$ is even more remarkable, as shown in Fig. 2(c). The overall magnitude of the torkance is reduced since fewer spin-orbit coupled scattering events take place. Remarkably, $\delta S_{x}$ (the main panel) is much larger than $\delta S_{y}$ (the inset), indicating that spin swapping dominates over the spin Hall effect, consistent with the results of Ref. [28]. Phase coherence results in quantum oscillations of both $\delta S_{x}$ and $\delta S_{y}$ (the inset). Nevertheless, while the oscillations of $\delta S_{y}$ in the normal

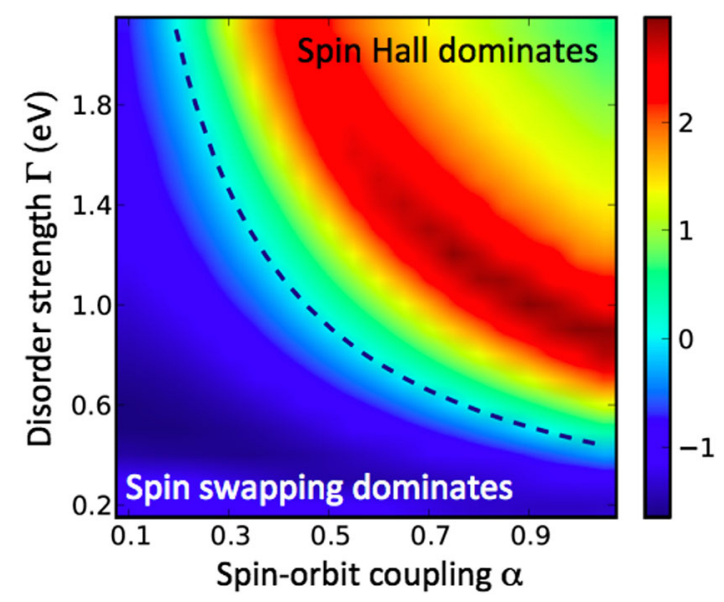

FIG. 3. Ratio between the magnitude of the fieldlike torque and dampinglike torque, $\tau_{\mathrm{DL}} / \tau_{\mathrm{FL}}$, as a function of $\Gamma$ and $\alpha$. The ratio is given in logarithmic scale and the dashed line indicates $\tau_{\mathrm{DL}} / \tau_{\mathrm{FL}}=1$. The parameters are the same as in Fig. 2 . metal are symmetric with respect to the center of the layer (a reminiscence of the standing nature of the wave functions), thereby resulting in a vanishing spin current injection, the oscillations of $\delta S_{x}$ are distorted and result in an effective spin current injection into the adjacent ferromagnet. As a consequence, the torkance is dominated by the fieldlike component, $\tau_{\mathrm{FL}}$, i.e., $\sim \mathbf{m} \times\left(\mathbf{z} \times \mathbf{j}_{e}\right)$ [the right panel of Fig. 2(d)] for all $\alpha$ 's, in agreement with the phenomenological discussion provided above.

In Fig. 3, the ratio $\tau_{\mathrm{DL}} / \tau_{\mathrm{FL}}$ is displayed as a function of disorder and spin-orbit coupling strengths. We find that the torque is dominated by the fieldlike component in the weak disorder or weak spin-orbit coupling regime, while it is dominated by the dampinglike component in the strong disorder or strong spin-orbit coupling regime. These different phases can be directly attributed to the spatial dependences shown in Fig. 2. When spin Hall effect dominates (the strong disorder regime), the torque is mostly dampinglike, $\tau_{\mathrm{DL}}>\tau_{\mathrm{FL}}$, and when spin swapping dominates (the weak disorder, Knudsen regime), the torque is mostly fieldlike, $\tau_{\mathrm{FL}}>\tau_{\mathrm{DL}}$. This behavior has been reproduced by varying the thickness of the normal metal while keeping the disorder fixed (not shown). These simulations demonstrate that, in ultrathin bilayers, fieldlike torques not only arise from the inverse spin galvanic effect-they can also emerge due to spin-dependent scattering in the normal metal. A necessary condition is that the thickness of the normal metal ought to be of the order of the mean free path $\left(d_{N} \sim \lambda\right)$.

Drift-diffusion model.-To prove that spin swapping fieldlike torque only occurs in the Knudsen regime and vanishes with strong disorder, we explicitly derive the spin swapping torque in the diffusive regime $\left(d_{N} \gg \lambda\right)$. The spin-orbit coupled spin transport in the normal metal can be modeled using the spin-charge diffusion equation developed in Ref. [32] in the first Born approximation,

$$
\begin{gathered}
e \mathbf{j}_{e} / \sigma_{N}=-\nabla \mu_{c}+\frac{\alpha_{\mathrm{sh}}}{2} \nabla \times \boldsymbol{\mu}, \\
e^{2} \mathcal{J}_{s}^{i} / \sigma_{N}=-\nabla \frac{\mu_{i}}{2}+\alpha_{\mathrm{sh}} \mathbf{e}_{i} \times \nabla \mu_{c}-\frac{\alpha_{\mathrm{sw}}}{2} \mathbf{e}_{i} \times(\boldsymbol{\nabla} \times \boldsymbol{\mu}),
\end{gathered}
$$

where $\sigma_{N}$ is the bulk conductivity, $\alpha_{\mathrm{sh}}=\alpha / \lambda k_{F}$ is the Hall angle from side jump scattering (within the first Born approximation, skew scattering is absent) and $\alpha_{\mathrm{sw}}=2 \alpha / 3$ is the spin swapping coefficient. $\lambda$ and $k_{F}$ are the mean free path and the Fermi wave vector, respectively. $\mu_{c}$ and $\boldsymbol{\mu}$ are the spin-independent and spin-dependent electrochemical potentials, related to the charge and spin accumulations by $\mu_{c}=n / \mathcal{N}$ and $\boldsymbol{\mu}=\delta \mathbf{S} / \mathcal{N}$, and $\mathcal{N}$ is the density of state. $\mathbf{j}_{e}$ is the current density vector, and $\mathcal{J}_{s, j}^{i}$ is the $i$ th spin component of the spin current flowing along the $j$ th direction. This set of equations is combined with the continuity equations $\boldsymbol{\nabla} \cdot \mathbf{j}_{e}=0$ and $\boldsymbol{\nabla} \cdot \mathcal{J}_{s}=-\boldsymbol{\mu}\left(\mathcal{N} / \tau_{\mathrm{sf}}\right)$, where $\tau_{\mathrm{sf}}$ is the spin relaxation time. The spin transport in the ferromagnet is modeled by similar drift-diffusion 
equations [31]. To model the torque exerted on the ferromagnet, we assume that the spin dephasing in the magnetic layer is so short that the incoming spin current is entirely absorbed within a few monolayers from the interface. The boundary conditions are then written [33]

$$
\begin{gathered}
j_{e, z}=2 g \Delta \mu_{c}+2 \gamma g \Delta \mu_{\|}, \\
\mathcal{J}_{s, z}^{\|}=2 \gamma g \Delta \mu_{c}+2 g \Delta \mu_{\|}, \\
\mathcal{J}_{s, z}^{\perp}=2\left(g_{r}^{\uparrow \downarrow} \Delta \mu_{\mathrm{op}}-g_{i}^{\uparrow \downarrow} \Delta \mu_{\mathrm{ip}}\right) \mathbf{m} \times \mathbf{y} \\
+2\left(g_{r}^{\uparrow \downarrow} \Delta \mu_{\mathrm{ip}}+g_{i}^{\uparrow \downarrow} \Delta \mu_{\mathrm{op}}\right) \mathbf{m} \times(\mathbf{y} \times \mathbf{m}),
\end{gathered}
$$

where $\mathcal{J}_{s, z}^{\|}=\mathcal{J}_{s, z} \cdot \mathbf{m}$ and $\mathcal{J}_{s, z}^{\perp}=\mathcal{J}_{s, z}-\mathcal{J}_{s, z}^{\|} \cdot \mathbf{m}$ is the spin current transverse to the magnetization $\mathbf{m}$. We define $g=\left(g_{\uparrow}+g_{\downarrow}\right) / 2$ and $\gamma=\left(g_{\uparrow}-g_{\downarrow}\right) / 2 g$, with $g_{s}$ being the interfacial conductance for spin $s$ and $g^{\uparrow \downarrow}=g_{r}^{\uparrow \downarrow}+i g_{i}^{\uparrow \downarrow}$ is the (complex) mixing conductance. The algebra to obtain the interfacial spin current is cumbersome but does not present technical difficulties (see Ref. [31] for details). We find that the torque possesses two contributions, $\boldsymbol{\tau}=\boldsymbol{\tau}_{\mathrm{sh}}+\boldsymbol{\tau}_{\mathrm{sw}}$, associated with spin Hall and spin swapping, respectively, and

$$
\boldsymbol{\tau}_{\mathrm{sh}}=\frac{\tilde{\alpha}_{\mathrm{sh}} j_{N}}{D_{\theta}} \eta_{0}\left[-\left(\tilde{g}_{r}^{\uparrow \downarrow}+\left|\tilde{g}^{\uparrow \downarrow}\right|^{2}\right) \mathbf{m} \times(\mathbf{y} \times \mathbf{m})+\tilde{g}_{i}^{\uparrow \downarrow} \mathbf{m} \times \mathbf{y}\right],
$$

$$
\begin{gathered}
\boldsymbol{\tau}_{\mathrm{sw}}=\alpha_{\mathrm{sw}} \frac{\tilde{\alpha}_{\mathrm{sh}} j_{N}}{D_{\theta}}\left[\left(\left|\tilde{g}^{\uparrow \downarrow}\right|^{2}-\eta \tilde{g}_{r}^{\uparrow \downarrow}\right) m_{z} \mathbf{m} \times \mathbf{x}\right. \\
\left.+\eta \tilde{g}_{i}^{\uparrow \downarrow} m_{z} \mathbf{m} \times(\mathbf{x} \times \mathbf{m})\right], \\
D_{\theta}=\eta_{0}\left[\left(\tilde{g}_{i}^{\uparrow \downarrow}\right)^{2}+\left(1+\tilde{g}_{r}^{\uparrow \downarrow}\right)\left(1+\tilde{g}_{r}^{\uparrow \downarrow}+\alpha_{\mathrm{sw}}\right)\right] \\
-\alpha_{\mathrm{sw}} m_{z}^{2}\left[\left(\tilde{g}_{i}^{\uparrow \downarrow}\right)^{2}+\left(\tilde{g}_{r}^{\uparrow \downarrow}-\eta\right)\left(1+\tilde{g}_{r}^{\uparrow \downarrow}+\alpha_{\mathrm{sw}}\right)\right] .
\end{gathered}
$$

Here $\sigma_{F, N}$ and $\lambda_{\mathrm{sf}}^{F, N}$ are the conductivity and spin diffusion length of the ferromagnetic (normal metal) layer, and $j_{N}$ is the charge current density flowing in the normal metal. In order to keep the notation compact, we defined the effective spin Hall angle $\tilde{\alpha}_{\mathrm{sh}}=\alpha_{\mathrm{sh}}\left(1-\cosh ^{-1} d_{N} / \lambda_{\mathrm{sf}}^{N}\right)$ and normalized mixing conductances $\tilde{g}_{j}^{\uparrow \downarrow}=4 \tilde{\lambda}_{\mathrm{sf}}^{N} g_{j}^{\uparrow \downarrow} / \sigma_{N}$, where $\tilde{\lambda}_{\mathrm{sf}}^{N}=\lambda_{\mathrm{sf}}^{N} / \tanh \left(d_{N} / \lambda_{\mathrm{sf}}^{N}\right)$ is the effective spin diffusion length of the normal metal. Finally, $\eta=\left(4\left(1-\gamma^{2}\right) g \tilde{\lambda}_{\mathrm{sh}}^{N} /\right.$ $\left.\sigma_{N} / 1+4\left(1-\gamma^{2}\right) g \tilde{\lambda}_{\mathrm{sf}}^{F} / \sigma_{F}\right)$, and $\eta_{0}=1+\eta+\alpha_{\mathrm{sw}}$.

The spin Hall torque, $\boldsymbol{\tau}_{\mathrm{sh}} \propto \alpha_{\mathrm{sh}}$ [Eq. (6)], produces the regular dampinglike torque $\mathbf{m} \times(\mathbf{y} \times \mathbf{m})$, with a small contribution to the fieldlike torque $\mathbf{m} \times \mathbf{y}$ [21]. These two torques are renormalized by the denominator $D_{\theta}$ that depends on $m_{z}^{2}$ through the spin swapping coefficient $\alpha_{\mathrm{sw}}$. More interestingly, the spin swapping torque, $\boldsymbol{\tau}_{\mathrm{sw}}$ [Eq. (7)], arises from the interplay between spin swapping and the spin Hall effect $\left(\propto \alpha_{\mathrm{sw}} \alpha_{\mathrm{sh}}\right)$ and is therefore at the second order in spin-orbit coupling. Furthermore, it generates two additional torque components: a dominating dampinglike torque $\left[\sim \eta \tilde{g}_{r}^{\uparrow \downarrow} m_{z} \mathbf{m} \times \mathbf{x}\right]$ and a small fieldlike torque $\left[\sim \tilde{g}_{i}^{\uparrow \downarrow} m_{z} \mathbf{m} \times(\mathbf{x} \times \mathbf{m})\right]$. In summary, since $g_{r}^{\uparrow \downarrow} \gg g_{i}^{\uparrow \downarrow}$, the spin torque in diffusive regime is always dominated by a dampinglike component and the fieldlike torque is vanishingly small, in agreement with our tightbinding calculations. The spin swapping induced fieldlike torque only emerges in the Knudsen regime, when semiclassical size effects are large.

Discussion and perspectives.-These results have interesting implications for experiments on spin-orbit torque $[13,14]$, spin pumping [11], and spin Seebeck [12]. In fact, most of these experiments are conducted on multilayers with thicknesses from $10 \mathrm{~nm}$ down to less than $1 \mathrm{~nm}$ [22-24,34]. In sputtered thin films the grain size ranges from 5 to $10 \mathrm{~nm}$, which implies that the transport is not diffusive and that extrinsic spin swapping can lead to sizable fieldlike torque, even in the absence of interfacial inverse spin galvanic effect. In addition, our numerical calculations disregard the effect of intrinsic (Berry phaseinduced) spin Hall effect, dominant in $4 d$ and $5 d$ transition metals. Then, one can reasonably expect that the spin-orbit coupling in the band structure should also induce spin swapping [35]. This effect is well known in semiconductors where the coherent precession about the local spin-orbit field induces, e.g., D'yakonov-Perel spin relaxation. Intrinsic spin swapping can be estimated using ab initio calculations, but it may be difficult to disentangle it from interfacial inverse spin galvanic effect [25].

To test the physics of spin swapping, one needs a magnetic stack that combines (i) an absence of interfacial spin-orbit coupling to quench the inverse spin galvanic effect, (ii) a long mean free path enabling semiclassical size effects, and (iii) strong bulk spin-orbit coupled spin scattering. A system that gathers these features is a magnetic bilayer involving a light metal doped with heavy elements, such as $\mathrm{Cu}(\mathrm{Bi}), \mathrm{Cu}(\mathrm{Ir})$, or $\mathrm{Ag}(\mathrm{Au})$. These materials display large extrinsic spin-orbit coupled scattering [36], and hence extrinsic spin swapping, together with ensuring the absence of interfacial spin-orbit coupling. Upon varying the thickness of the metallic layer from below to above the mean free path, a substantial modulation of the fieldlike torque is expected.

We conclude this Letter by commenting on the impact of spin swapping on spin pumping, the Onsager reciprocal of spin transfer torque. When excited, a precessing magnetization pumps a spin current, polarized along $\sim \mathbf{m} \times \partial_{t} \mathbf{m}$, into the normal metal [37]. Such a spin current can be converted into a charge current through the inverse spin Hall effect [11], but it also enhances the magnetic damping of the ferromagnet [37]. Upon spin swapping, this pumped spin current is converted into another spin current polarized along $\mathbf{y} \times\left(\mathbf{m} \times \partial_{t} \mathbf{m}\right)$ (with $\mathbf{y}$ being the direction of the spin-orbit field perpendicular to the scattering plane). While this new spin current does not contribute to 
additional electric signals, it produces an anisotropic damping on the form $\sim m_{y} \partial_{t} \mathbf{m}$. This effect vanishes by symmetry in homogeneous ferromagnets, but it is expected to survive in magnetic domain walls resulting in unconventional magnetic damping. Finally, spin swapping is expected to induce additional magnetoresistive effects in the Knudsen regime. Further theoretical investigations and experimental explorations are necessary for uncovering the full implications of these phenomena.

A. M. acknowledges the inspiring discussions with T. Valet and H. B. M. S. thanks S. Feki and B. Hadri for their valuable technical support. This work was supported by the King Abdullah University of Science and Technology (KAUST).

*aurelien.manchon@kaust.edu.sa †hamed.saidaoui@gmail.com

[1] T. Jungwirth, J. Wunderlich, and K. Olejnk, Nat. Mater. 11, 382 (2012).

[2] A. Manchon, H. C. Koo, J. Nitta, S. Frolov, and R. A. Duine, Nat. Mater. 14, 871 (2015).

[3] E. L. Ivchenko and G. E. Pikus, Pis'ma Zh. Eksp. Teor. Fiz. 27, 640 (1978) [JETP Lett. 27, 604 (1978)].

[4] G. Vignale, J. Supercond. Novel Magn. 23, 3 (2010).

[5] M. I. Dyakonov and V. I. Perel, Phys. Lett. 35A, 459 (1971).

[6] S. Murakami, N. Nagaosa, and S.-C. Zhang, Science 301, 1348 (2003); J. Sinova, D. Culcer, Q. Niu, N. A. Sinitsyn, T. Jungwirth, and A. H. MacDonald, Phys. Rev. Lett. 92, 126603 (2004).

[7] I. A. Ado, I. A. Dmitriev, P. M. Ostrovsky, and M. Titov, Europhys. Lett. 111, 37004 (2015).

[8] L. Vila, T. Kimura, and Y. C. Otani, Phys. Rev. Lett. 99, 226604 (2007).

[9] C. Du, H. Wang, F. Yang, and P. C. Hammel, Phys. Rev. B 90, 140407 (2014).

[10] X. Wang, J. Xiao, A. Manchon, and S. Maekawa, arXiv: 1407.8278 .

[11] E. Saitoh, M. Ueda, H. Miyajima, and G. Tatara, Appl. Phys. Lett. 88, 182509 (2006).

[12] K. Uchida et al., Nat. Mater. 9, 894 (2010).

[13] I. M. Miron, K. Garello, G. Gaudin, P.-J. Zermatten, M. V. Costache, S. Auffret, S. Bandiera, B. Rodmacq, A. Schuhl, and P. Gambardella, Nature (London) 476, 189 (2011).

[14] L. Liu, C.-F. Pai, Y. Li, H. W. Tseng, D. C. Ralph, and R. A. Buhrman, Science 336, 555 (2012).

[15] B. A. Bernevig and O. Vafek, Phys. Rev. B 72, 033203 (2005); I. Garate and A. H. MacDonald, Phys. Rev. B 80, 134403 (2009).

[16] A. Manchon and S. Zhang, Phys. Rev. B 78, 212405 (2008); 79, 094422 (2009).

[17] J. Železný, H. Gao, K. Výborný, J. Zemen, J. Mašek, A. Manchon, J. Wunderlich, J. Sinova, and T. Jungwirth Phys. Rev. Lett. 113, 157201 (2014).

[18] A. Brataas and K. Hals, Nat. Nanotechnol. 9, 86 (2014).

[19] X. Qiu, K. Narayanapillai, Y. Wu, P. Deorani, D.-H. Yang, W.-S. Noh, J.-H. Park, K.-J. Lee, H.-W. Lee, and H. Yang, Nat. Nanotechnol. 10, 333 (2015).
[20] A. R. Mellnik et al., Nature (London) 511, 449 (2014); Y. Fan et al., Nat. Mater. 13, 699 (2014).

[21] P. M. Haney, H.-W. Lee, K.-J. Lee, A. Manchon, and M. D. Stiles, Phys. Rev. B 87, 174411 (2013).

[22] X. Fan, J. Wu, Y. Chen, M. J. Jerry, H. Zhang, and J. Q. Xiao, Nat. Commun. 4, 1799 (2012); C.-F. Pai, M.-H. Nguyen, C. Belvin, L. H. Vilela-Leão, D. C. Ralph, and R. A. Buhrman, Appl. Phys. Lett. 104, 082407 (2014).

[23] J. Kim, J. Sinha, M. Hayashi, M. Yamanouchi, S. Fukami, T. Suzuki, S. Mitani, and H. Ohno, Nat. Mater. 12, 240 (2012); J. Kim, J. Sinha, S. Mitani, M. Hayashi, S. Takahashi, S. Maekawa, M. Yamanouchi, and H. Ohno, Phys. Rev. B 89, 174424 (2014).

[24] M.-H. Nguyen, D. C. Ralph, and R. A. Buhrman, Phys. Rev. Lett. 116, 126601 (2016).

[25] F. Freimuth, S. Blügel, and Y. Mokrousov, Phys. Rev. B 90, 174423 (2014); P. M. Haney, H.-W. Lee, K.-J. Lee, A. Manchon, and M.D. Stiles, Phys. Rev. B 88, 214417 (2013).

[26] X. Wang and A. Manchon, Phys. Rev. Lett. 108, 117201 (2012); H. Li et al., Phys. Rev. B 91, 134402 (2015); K.-S. Lee, D. Go, A. Manchon, P. M. Haney, M. D. Stiles, H.-W. Lee, and K.-J. Lee, Phys. Rev. B 91, 144401 (2015).

[27] M. B. Lifshits and M. I. Dyakonov, Phys. Rev. Lett. 103, 186601 (2009).

[28] H. B. M. Saidaoui, Y. Otani, and A. Manchon, Phys. Rev. B 92, 024417 (2015).

[29] C. W. Groth, M. Wimmer, A. R. Akhmerov, and X. Waintal, New J. Phys. 16, 063065 (2014).

[30] The magnitude of the torkance reported in Fig. 2(b), $\tau \sim 10^{-2}(h / e)$ corresponds to an effective field of $h / j_{e}=\tau /\left(M_{s} d\right) \sim 100 \mathrm{Oe} /\left(\mathrm{A} / \mathrm{cm}^{2}\right)$ for a ferromagnet of thickness $d=1 \mathrm{~nm}$ and saturation magnetization of $M_{s}=10^{6} \mathrm{~A} / \mathrm{m}$. The large reduction of the torkance in the weak disorder regime is partly due to the absence of interfacial scattering in our simulation of the size effect regime.

[31] See Supplemental Material at http://link.aps.org/ supplemental/10.1103/PhysRevLett.117.036601 for the two dimensional mapping of the spin density, as well as the derivation of Eqs. (6) and (7).

[32] R. V. Shchelushkin and Arne Brataas, Phys. Rev. B 71, 045123 (2005).

[33] A. Brataas, G. E. W. Bauer, and P. J. Kelly, Phys. Rep. 427, 157 (2006).

[34] C. O. Avci et al., Phys. Rev. B 89, 214419 (2014); C.-F. Pai, Y. Ou, L. H. Villela-Leão, D. C. Ralph, and R. A. Buhrman, Phys. Rev. B 92, 064426 (2015).

[35] S. Sadjina, A. Brataas, and A. G. Malshukov, Phys. Rev. B 85, 115306 (2012).

[36] Y. Niimi, M. Morota, D. H. Wei, C. Deranlot, M. Basletic, A. Hamzic, A. Fert, and Y. Otani, Phys. Rev. Lett. 106, 126601 (2011); Y. Niimi, Y. Kawanishi, D. H. Wei, C. Deranlot, H. X. Yang, M. Chshiev, T. Valet, A. Fert, and Y. Otani, Phys. Rev. Lett. 109, 156602 (2012); S. Lowitzer, M. Gradhand, D. Ködderitzsch, D. V. Fedorov, I. Mertig, and H. Ebert, Phys. Rev. Lett. 106, 056601 (2011).

[37] Y. Tserkovnyak, A. Brataas, and G. E. W. Bauer, Phys. Rev. Lett. 88, 117601 (2002). 